\title{
A New Theory of Dynamic Arrest in Colloidal Mixtures
}

\author{
R. Juárez-Maldonado and M. Medina-Noyola \\ Instituto de Física "Manuel Sandoval Vallarta", \\ Universidad Autónoma de San Luis Potosí, \\ Álvaro Obregón 64, 78000 San Luis Potosí, SLP, México
}

(Dated: November 1, 2018)

\begin{abstract}
We present a new first-principles theory of dynamic arrest in colloidal mixtures based on the multi-component self-consistent generalized Langevin equation (SCGLE) theory of colloid dynamics [Phys. Rev. E 72, 031107 (2005); ibid 76, 039902 (2007)]. We illustrate its application with the description of dynamic arrest in two simple model colloidal mixtures, namely, the hard-sphere and the repulsive Yukawa binary mixtures. Our results include the observation of the two patterns of dynamic arrest, one in which both species become simultaneously arrested, and the other involving the sequential arrest of the two species. The latter case gives rise to mixed states in which one species is arrested while the other species remains mobile. We also derive the ("fixed point") equations for the non-ergodic parameters of the system, which takes the surprisingly simple form of a system of coupled equations for the localization length of the particles of each species. The solution of this system of equations indicates unambiguously which species is arrested (finite localization length) and which species remains ergodic (infinite localization length). As a result, we are able to draw the entire ergodic-non-ergodic phase diagram of the binary hard-sphere mixture.

PACS numbers: 64.70.Pf, 61.20.Gy, 47.57.J-
\end{abstract}




\section{INTRODUCTION}

The fundamental understanding of dynamically arrested states is a major challenge of contemporary statistical physics and materials science [1, 2, 3]. Model experimental colloidal suspensions, whose dynamics has been the subject of study on its own right [4, 5, 6], have played an essential role in the study of dynamic arrest phenomena, providing experimental realizations in finely-controlled systems and conditions [7, 8, 9, 10, 11]. Thus, it is an experimental fact that by changing a macroscopic control parameter such as the volume fraction, or by tuning the effective inter-particle interactions, one may drive the system from the region of equilibrium (ergodic) states to the region of dynamically arrested (non-ergodic) states. First-principles models and theories that predict and describe these transitions thus constitute an essential aspect of the fundamental understanding of these phenomena. The mode coupling theory (MCT) of the ideal glass transition [3, 12] is perhaps the most comprehensive theory of this sort, some of whose predictions have found beautiful experimental confirmation [7, 10, 11]. Many issues, however, still remain to be understood, and this has

prompted the proposal of extended versions of this theory [13, 14, 15], or the development of alternative approaches [16].

In this context, in recent work a new first-principles theory of dynamic arrest has been proposed [17, 18, 19] which is essentially the application of the self-consistent generalized Langevin equation (SCGLE) theory of colloid dynamics [20, 21, 22, 23] to the analysis of dynamic arrest phenomena in colloidal systems. The SCGLE theory was originally devised to describe the tracer and collective diffusion properties of colloidal dispersions in the short- and intermediate-times regimes. Its self-consistent character, however, introduces a non-linear dynamic feedback, leading to the prediction of dynamic arrest in these systems, similar to that exhibited by the mode coupling (MC) theory of the ideal glass transition. The resulting theory of dynamic arrest in colloidal dispersions was applied in recent work to describe the glass transition in three mono-disperse experimental systems with specific (hard-sphere, screened electrostatic, and depletion) inter-particle effective forces [17, 18], with the same quantitative predictive power as conventional MCT, but with a smaller degree of difficulty in its application [19]. The present paper introduces the multi-component extension of the SCGLE theory of colloid dynamics [24, 25] as a new alternative first-principles theory of dynamic arrest in colloidal mixtures. 
Colloidal mixtures offer a richer variety of possible dynamic arrest scenarios [26, 27, 28, 29, 30, 31]. These include, for example, the possibility that the particles of one species become arrested, while the particles of the other species continue to diffuse through the disordered matrix of the arrested particles. In fact, an important prediction of the multi-component extension of MCT [32, 33] was precisely the existence of these mixed states [34, 35, 36]. Later studies of dynamic arrest in mixtures [37, 38, 39, 40, 41, 42] have been based on MCT not because it is the ultimate and definitive theory, but because it was virtually the only first-principles theory of dynamic arrest available that had a high level of predictive capability. However, relevant issues remain that have not been resolved by its application. For example, although the one-component version of MCT predicts the existence of repulsive glasses in a hard-sphere system, and its melting upon the addition of a small amount of smaller particles (depletion forces), followed by the reentrance to a new kind of (attractive) glass [10], no reentrance seems to be predicted by the two-component version of MCT [43]. Issues like this merit the development of alternative approaches to the description of dynamic arrest phenomena in mixtures, particularly if they are independent from conventional MCT. Although the SCGLE theory presented here has several features in common with MCT, such as its use of exact memory function expressions for the concentration-concentration time-correlation functions, in the SCGLE theory the derivations, approximations and argumentation are conceived and based on a totally different fundamental and conceptual framework [20, 21, 22, 23, 24, 25].

Besides presenting the SCGLE theory of dynamic arrest in colloid mixtures, in the present paper we illustrate the scenarios predicted by this theory in the simplest conditions, namely, in its application to model binary mixtures. For two such systems, a repulsive Yukawa mixture and a hard-sphere mixture, we numerically solve the full self consistent system of dynamic equations for the so-called propagators (or Green's functions) of the self and collective intermediate scattering functions. We illustrate in this manner the two possible dynamic arrest patterns possible in a binary colloidal mixture, namely, the simultaneous and the sequential arrest of the two species. In the latter case, we discuss the hybrid or partially-arrested states in which one species is dynamically arrested while the other species remains ergodic.

Determining the location of the ergodic-non-ergodic transitions and calculating the nonergodic parameters may be done by solving the self-consistent system of equation or, more 
simply, by finding the long-time asymptotic stationary ("fixed-point") solutions of the selfconsistent system of equations. Here we also address this issue, and report the derivation of a strikingly simple and general result which takes the form of a system of coupled equations for the localization length of the particles of each species. Solving this system of equations leads to the unambiguous identification of which species is arrested (finite localization length) and which species remains ergodic (infinite localization length). As a result, we are able to draw the entire ergodic-non-ergodic phase diagram of the binary hard-sphere mixture, which is one of the main specific contributions of this work.

The present paper starts with a brief summary of the SCGLE theory of the dynamics of colloidal mixtures. This theory was developed in Ref. [25] and tested there through its comparison with the short- and intermediate-time results of Brownian dynamics computer simulations for a model colloidal mixture interacting through screened Coulomb pair interactions. That original version of the SCGLE theory required as an input not only the partial static structure factors, but also other equilibrium static structural information associated with the exact short-time conditions that this initial version of the theory aimed at incorporating. As it has been shown more recently [19], however, a simplified version of the SCGLE theory, in which this exact short-time information is neglected, happens to be equally accurate, particularly in the long-time regime and with respect to the predicted dynamic arrest scenarios. Thus, we shall refer to this simplified version simply as the SCGLE theory, which we review in the following section.

Although our main interest here is in the description of dynamic arrest phenomena, in section III we start our discussion by reviewing the predictive capability of this simplified theory in the short- and intermediate-time regimes of the model binary Yukawa mixture studied in Ref. [25] in one of the fully ergodic states considered in that work. We then proceed to illustrate the dynamic arrest scenarios predicted by this theory, by taking as the initial reference the same repulsive Yukawa mixture, and increase the intensity of the repulsion parameter (a process that amounts to effectively lowering the temperature), until reaching a transition to a dynamically arrested state. This turns out to be a transition from a fully ergodic to a fully arrested state in which both species are simultaneously arrested. In the second of our illustrative examples, in section IV we consider a hard-sphere model colloidal mixture, whose state parameters are the size disparity and the concentration of the two species. Here we present an example of a transition to a partially arrested state, 
in which the large particles become arrested and the smaller particles continue to diffuse in the disordered matrix formed by the arrested species. In section $\mathrm{V}$ we derive the equations for the non-ergodic parameters of the system, and illustrate its use in the context of the hard-sphere binary mixture by determining its entire dynamic-arrest phase diagram. The main conclusions of this paper are finally summarized in section VI.

\section{THE SCGLE THEORY OF THE DYNAMICS OF COLLOIDAL MIXTURES}

In this section we summarize the main elements of the self- consistent theory of the dynamics of colloidal mixtures presented in Refs. [24, 25]. For a colloidal mixture with $\nu$ species, the dynamic properties can be described in terms of the relaxation of the fluctuations $\delta n_{\alpha}(\mathbf{r}, t)$ of the local concentration $n_{\alpha}(\mathbf{r}, t)$ of colloidal particles of species $\alpha(=1,2, \ldots \nu)$ around its bulk equilibrium value $n_{\alpha}$. The average decay of $\delta n_{\alpha}(\mathbf{r}, t)$ is described by the timedependent correlation function $F_{\alpha \beta}(k, t) \equiv\left\langle\delta n_{\alpha}(\mathbf{k}, t) \delta n_{\beta}(-\mathbf{k}, 0)\right\rangle$ of the Fourier transform $\delta n_{\alpha}(\mathbf{k}, t) \equiv\left(1 / \sqrt{N_{\alpha}}\right) \sum_{i=1}^{N} \exp \left[i \mathbf{k} \cdot \mathbf{r}_{\mathbf{i}}^{(\alpha)}(t)\right]$ of the fluctuations $\delta n_{\alpha}(\mathbf{r}, t)$, with $\mathbf{r}_{\mathbf{i}}^{(\alpha)}(t)$ being the position of particle $i$ of species $\alpha$ at time $t . F_{\alpha \beta}(k, t)$ is referred to as the partial intermediate scattering function, measured by experimental techniques such as dynamic light scattering [4, 6]. One can also define the self component of $F_{\alpha \beta}(k, t)$, referred to as the self-intermediate scattering function, as $F_{\alpha \beta}^{(s)}(k, t) \equiv \delta_{\alpha \beta}\left\langle\exp \left[i \mathbf{k} \cdot \Delta \mathbf{R}^{(\alpha)}(t)\right]\right\rangle$, where $\Delta \mathbf{R}^{(\alpha)}(t)$ is the displacement of any of the $N_{\alpha}$ particles of species $\alpha$ over a time $t$, and $\delta_{\alpha \beta}$ is Kronecker's delta function.

For notational economy, let us denote the matrix with elements $F_{\alpha \beta}(k, t)(\alpha, \beta=$ $1,2, \ldots, \nu)$ by $F(k, t)$ or simply by $F(t)$, and similarly for $F^{(s)}(t)$. These matrices have the initial values $F(t=0)=S$ and $F^{(s)}(t=0)=I$, where $I$ is the identity matrix, $I_{\alpha \beta} \equiv \delta_{\alpha \beta}$, and the elements of the matrix $S$ are the partial static structure factors, $S_{\alpha \beta}(k) \equiv\left\langle\delta n_{\alpha}(\mathbf{k}, 0) \delta n_{\beta}(-\mathbf{k}, 0)\right\rangle$. The matrix of intermediate scattering functions may also be normalized by its initial value, to define the collective propagator matrix $\Psi(t) \equiv F(t) S^{-1}$, which is such that $\Psi(0)=I$. In a similar manner, we define the self propagator matrix as $\Psi^{(s)}(t) \equiv F^{(s)}(t)$, normalized to $\Psi^{(s)}(0)=I$.

The multi-component version of the SCGLE theory starts with the exact time-evolution equation that governs the relaxation of the Fourier transform $(\mathrm{FT}) \delta n_{\alpha}(\mathbf{k}, t)$ of the fluctuations of the local concentration of species $\alpha$. This equation also governs the relaxation 
of the partial intermediate scattering functions $F_{\alpha \beta}(k, t)$, and can be written as an exact expression for the Laplace transform (LT) $F(z)$ of the matrix $F(t)$, namely [24],

$$
F(z)=\left\{z+(I+C(z))^{-1} k^{2} D S^{-1}\right\}^{-1} S
$$

where the elements of the matrix $D$ are given by $D_{\alpha \beta} \equiv \delta_{\alpha \beta} D_{\alpha}^{0}$, with $D_{\alpha}^{0}$ being the diffusion coefficient of species $\alpha$ in the absence of interactions. This is related with the solvent friction coefficient on an isolated particle of species $\alpha, \zeta_{\alpha}^{0}$, through the Einstein relation, $D_{\alpha}^{0} \equiv k_{B} T / \zeta_{\alpha}^{0}$. The elements $C_{\alpha \beta}(k, z)$ of the matrix $C(z)$ are the LT of the so-called irreducible memory functions $C_{\alpha \beta}(k, t)$ [36, 44]. The corresponding result for the "self" component, $F^{(s)}(z)$, of the matrix $F(z)$ reads

$$
F^{(s)}(z)=\left\{z+\left(I+C^{(s)}(z)\right)^{-1} k^{2} D\right\}^{-1}
$$

where the matrix $C^{(s)}(z)$ is the corresponding irreducible memory function.

The second ingredient of the SCGLE theory is the intuitive notion that collective and self dynamics may be connected in a simple manner. Vineyard's approximation, in which $\Psi(t)$ is approximated by $\Psi^{(s)}(t)$, is a rather primitive implementation of this idea. The SCGLE theory incorporates the same notion, but implemented at the level of the irreducible memory functions $C(t)$ and $C^{(s)}(t)$, namely,

$$
C(t)=C^{(s)}(t)
$$

which we shall simply refer as the Vineyard-like approximation. Let us mention that in the original proposal of the multi-component version of the SCGLE theory [25] the difference $\left[C(t)-C^{(s)}(t)\right]$ was approximated not by zero, as in this equation, but by its exact ("single exponential") short-time limit $\left[C^{S E X P}(t)-C^{(s), S E X P}(t)\right]$, given the fact that explicit expressions exist [24, 45] for the short-time limits $C^{S E X P}(t)$ and $C^{(s), S E X P}(t)$ of the irreducible memory functions $C(t)$ and $C^{(s)}(t)$ in terms of equilibrium static structural properties of the system, which are considered known. More recently, however, it was found [19] that an equally accurate but much simpler approximation results if the difference $\left[C(t)-C^{(s)}(t)\right]$ is approximated by zero, as in Eq. (2.3), thus eliminating the need to determine the short-time memory functions $C^{S E X P}(t)$ and $C^{(s), S E X P}(t)$.

The third ingredient of the SCGLE theory was suggested [25] as the interpolation of 
$C^{(s)}(t)$ between its two exact limits at small and large wave-vectors, by means of a phenomenological but universal interpolation device. We know that the $C^{(s), S E X P}(t)$ is also the exact large- $k$ limit of $C^{(s)}(t)$, whereas the small- $k$ limit is the so-called time-dependent friction function matrix $\Delta \zeta^{*}(t)$. Thus, we write $C^{(s)}(k, t)=C^{(s), S E X P}(k, t)+\left[\Delta \zeta^{*}(t)-\right.$ $\left.C^{(s), \operatorname{SEXP}}(k, t)\right] \lambda(k)$, where we have written explicitly the $k$-dependence of the various matrices. The matrix $\lambda$ is a matrix of phenomenological interpolating functions, whose elements are defined as [22, 25]

$$
\lambda_{\alpha \beta}(k) \equiv \delta_{\alpha \beta} /\left[1+\left(k / k_{\min }^{(\alpha)}\right)^{2}\right],
$$

with $k_{\min }^{(\alpha)}$ being the position of the first minimum following immediately the main peak of the partial static structure factor $S_{\alpha \alpha}(k)$. At times long enough that the short-time memory function $C^{(s), \operatorname{SEXP}}(k, t)$ has already decayed, the interpolation formula above for $C^{s}(k, t)$ simplifies still further, reading

$$
C^{(s)}(k, t)=\left[\Delta \zeta^{*}(t)\right] \lambda(k)
$$

As suggested in Ref. [19], it is this approximation that enters in the present formulation of the multi-component SCGLE theory of dynamic arrest.

The matrix $\Delta \zeta^{*}(t)$ in eq. (2.5) is a diagonal matrix whose $\alpha$ th diagonal element, $\Delta \zeta_{\alpha}^{*}(t)$, is the time-dependent friction function of particles of species $\alpha$. The final ingredient of the SCGLE theory is an approximate but general result for this property, which, as explained below, was derived as an application of the GLE formalism [46, 47, 48] to tracer diffusion phenomena. Such an expression reads [48]

$$
\Delta \zeta_{\alpha}^{*}(t)=\frac{D_{\alpha}^{0}}{3(2 \pi)^{3}} \int d^{3} k k^{2}\left[F^{(s)}(t)\right]_{\alpha \alpha}\left[c \sqrt{n} F(t) S^{-1} \sqrt{n} h\right]_{\alpha \alpha},
$$

with the elements of the $k$-dependent matrices $h$ and $c$ being the Fourier transforms $h_{\alpha \beta}(k)$ and $c_{\alpha \beta}(k)$ of the Ornstein-Zernike total and direct correlation functions, respectively. Thus, $h$ and $c$ are related to $S$ by $S=I+\sqrt{n} h \sqrt{n}=[I-\sqrt{n} c \sqrt{n}]^{-1}$, with the matrix $\sqrt{n}$ defined as $[\sqrt{n}]_{\alpha \beta} \equiv \delta_{\alpha \beta} \sqrt{n_{\alpha}}$.

Let us now comment on the origin of Eq. (2.6). In Ref. [46] the effective Langevin equation of a tracer colloidal particle interacting with the other particles of a mono-disperse suspension was derived, using the concept of contraction of the description [47] (a summary 
of such derivation is contained in Appendix B of Ref. [18]). Besides the solvent friction force, $-\zeta^{0} \mathbf{V}(t)$, the direct interactions of the tracer particle with the other particles give rise to an additional friction term of the form $-\int_{0}^{t} d t^{\prime} \Delta \zeta\left(t-t^{\prime}\right) \mathbf{V}\left(t^{\prime}\right)$, where $\mathbf{V}(t)$ is the tracer particle's velocity at time $t$. In the process, an exact result for the time-dependent friction function $\Delta \zeta^{*}(t) \equiv \Delta \zeta(t) / \zeta^{0}$ is generated which, when complemented with two well defined approximations, leads to the mono-disperse version of Eq. (2.6) . The multi-component extension was derived in Ref. [48] from the exact result in eq. (124) of that reference, upon the introduction of the "homogeneous fluid" and the "decoupling" approximations (eqs. (135)-(138) and eq. (144) of the same reference).

Finally, let us mention that eqs. (2.1) and (2.2) are exact results, and that eq. (2.6) derives from the exact result just referred to. Hence, it should not be a surprise that the same results are employed by other theories; in fact, these equations also appear in the formulation of MCT. The difference lies, of course, in the way we relate them and use them; in this sense, the distinctive elements of our theory are then the Vineyard-like approximation in eq. (2.3) and the factorization approximation in eq. (2.5).

\section{ILLUSTRATIVE APPLICATION: THE MODEL YUKAWA MIXTURE}

The set of coupled equations (2.1)-(2.6) constitutes the self-consistent generalized Langevin equation theory of the dynamics of colloidal mixtures, and has to be solved numerically. For this, equations (2.1) and (2.2) are first Laplace-inverted, and written as a set of coupled integro-differential equations involving functions of $k$ and $t$. These functions are then discretized in a mesh of points large enough to ensure independence of the solution with respect to the size of the mesh. The discretized system of equations is then solved by a straightforward direct iteration method or, for the long times required in the study of dynamic arrest phenomena, by the methods described in Refs. [49, 50].

To illustrate its quantitative accuracy, the SCGLE theory was applied in Ref. [25] to the calculation of the dynamic properties of a particular model system, namely, a binary mixture of Brownian particles interacting through a hard-core pair potential of diameter $\sigma$, assumed the same for both species, plus a repulsive Yukawa tail of the form (in units of the thermal energy $k_{B} T=\beta^{-1}$ ) 


$$
\beta u_{\alpha \beta}(r)=A_{\alpha} A_{\beta} \frac{e^{-z\left(\frac{r}{\sigma}-1\right)}}{r / \sigma} .
$$

The dimensionless parameters that define the thermodynamic state of this system are the total volume fraction $\phi \equiv \frac{\pi}{6} n \sigma^{3}$ (with $n$ being the total number concentration, $n=n_{1}+n_{2}$ ), the relative concentrations $x_{\alpha} \equiv \frac{n_{\alpha}}{n}(\alpha=1,2)$, and the potential parameters $A_{1}, A_{2}$, and z. As in Ref. [25], here we also assume that the free-diffusion coefficient $D_{\alpha}^{0}$ of both species are identical, i.e., $D_{1}^{0}=D_{2}^{0}=D^{0}$. Explicit values of the parameters $\sigma$ and $D^{0}$ are not needed, since the dimensionless dynamic properties, such as $F_{\alpha \beta}(k, t)$, only depend on the dimensionless parameters specified above, when expressed in terms of the scaled variables $k \sigma$, and $t / t_{0}$, where $t_{0} \equiv \sigma^{2} / D^{0}$. In Ref. [25] Brownian dynamics simulations were reported for the static and dynamic properties of this system with fixed screening parameter $z=0.15$ and various coupling parameters $A_{1}$ and $A_{2}$ and volume fractions, in the short- and intermediatetime regimes.

The partial static structure factors $S_{\alpha \beta}(k)$ obtained from those simulations are also employed here as the static input needed in the implementation of the SCGLE theory. As a result, we can calculate all the dynamic properties entering in the self-consistent equations above, and other properties that derive from them, such as the mean squared displacements $W_{\alpha}(t) \equiv<\left[\Delta \mathbf{R}^{(\alpha)}(t)\right]^{2}>/ 6$ or the time-dependent diffusion coefficients $D_{\alpha}(t) \equiv W_{\alpha}(t) / t$. In Fig. 1 a comparison of these results with the Brownian dynamics calculations is presented, involving the case of a binary mixture with $z=0.15, A_{1}=10$, and $A_{2}=10 \sqrt{5}$. The volume fraction of the more interacting species is kept fixed at $\phi_{2}=2.2 \times 10^{-4}$ and $\phi_{1}$ takes the values $\phi_{1}=0.725 \times 10^{-4}$ (left column), $\phi_{1}=2.2 \times 10^{-4}$ (middle column), and $\phi_{1}=6.6 \times 10^{-4}$ (right column), corresponding to molar fractions $x_{1}=0.25, x_{1}=0.5$ and $x_{1}=0.75$, respectively. This figure corresponds to the same conditions as Fig. 3 of Ref. [25], and illustrates the fact that the present simplified SCGLE theory also provides an excellent description of the relaxation of concentration fluctuations in colloidal suspensions in the short- and intermediate-time regimes illustrated in the figure. We notice that the largest departures of the theoretical results from the simulation data appear only at the short times illustrated by the time $t=t_{0}$, but the agreement improves at longer times, as illustrated by the time $t=10 t_{0}$. The main message of this figure is that the present self-consistent theory of colloid dynamics provides an adequate description of the dynamic properties of a simple idealized mixture also in the short- and intermediate-time regimes, even though it 
does not satisfy the exact short-time conditions built in the original proposal of this theory [25]. With this confidence, let us now explore the long-time regime under conditions typical of the vicinity of a transition to dynamically arrested states.

For this, let us take the conditions of the middle column of Fig. 1 as a reference, and let us keep the parameters $\phi_{1}, \phi_{2}$ and $z$ fixed $\left(\phi_{1}=\phi_{2}=2.2 \times 10^{-4}\right.$ and $\left.z=0.15\right)$ while we increase the coupling parameters $A_{1}$ and $A_{2}$ in an identical proportion, to mimic a process of lowering the temperature. Thus, let us write $A_{\alpha}=A_{\alpha}^{0} \chi(\alpha=1,2)$, with $A_{1}^{0}=10$ and $A_{2}^{0}=10 \sqrt{5}$, and increase $\chi$ from its unit value in Fig. 1 until a transition of dynamic arrest is encountered. In this case, we do not run computer simulations to provide the input partial static structure factors needed in the self-consistent scheme. Instead, we resort to an approximate liquid state theory, namely, the solution of the Ornstein-Zernike integral equation within the hyper-netted-chain (HNC) approximation [51]. In doing this we only loose quantitative precision, but no qualitative accuracy. In fact, the HNC approximation will yield the same scenario, but at effective values of the coupling parameters $A_{1}$ and $A_{2}$ larger than the actual ones. For example, the fitting curves for the partial static structure factors in the middle column of Fig. 1 were obtained using the HNC approximation with effective coupling parameters given by $A_{\alpha}=A_{\alpha}^{0} \chi$ with $\chi=1.225$.

In Fig. 2 we present the results of the SCGLE theory for the diagonal elements $\Psi_{\alpha \alpha}(k, t)$ of the collective propagator matrix $\Psi(t)$ as a function of time for fixed wave-vector $k=k_{\max }$, where $k_{\max }$ is the position of the main peak of the corresponding static structure factor $S_{\alpha \alpha}(k)$. This figure illustrates the fact that as we lower the temperature starting from the reference state $(\chi=1.225)$ the system reaches eventually a transition to an arrested state. This occurs for $A_{1}=42.57$, and $A_{2}=95.19$, i.e., for $\chi=4.257$, and this is a transition from a fully ergodic to a fully arrested state, i.e., both species become simultaneously arrested. This is evidenced by the fact that below this threshold condition, exemplified in the figure by the case $\chi=4.255$, both propagators decay to zero, whereas above this condition, exemplified by the case $\chi=4.330$, they decay to a finite non-ergodic value. Thus, the nonergodic parameters jump discontinuously at the transition, from zero in the ergodic side, to finite values in the glass side. Exactly the same behavior is exhibited by the self-diffusion propagators $\Psi_{11}^{(s)}(k, t)$ and $\Psi_{22}^{(s)}(k, t)$, not included in the present illustration. Let us stress that this process of increasing $A_{1}$ and $A_{2}$ keeping $z$ fixed is a mathematical, rather than physical, exercise; the practicable experimental manner to increase $A_{1}$ and $A_{2}$ would be to 
increase the particle's electric charge (or to lower the dielectric constant of the solvent), which in reality would also increase the screening parameter $z$. Here, however, we use this exercise only to illustrate the pattern of dynamic arrest transition in colloidal mixtures in which the particles of both species become localized simultaneously.

Similar calculations can be carried out for other regions of the parameter space $\left(\phi_{1}, \phi_{2}, A_{1}, A_{2}, z\right)$, but this scanning and its results in terms of other possible regions that exhibit interesting dynamic behavior constitutes an independent issue, beyond our current objective of introducing our SCGLE theory of dynamic arrest in mixtures and illustrating its use. We can mention, however, that in other regions of this state space the theory predicts also the other pattern of dynamic arrest, which we now illustrate in the context of a simpler system, namely, the hard-sphere colloidal mixture.

\section{THE HARD-SPHERE MIXTURE}

Let us now consider the binary hard spheres model dispersion, i.e., the uncharged version of the previous system in which we now allow for hard-sphere size disparity. Thus, we consider two species of particles of hard core diameters $\sigma_{1}$ and $\sigma_{2}$, present at concentrations $n_{1}$ and $n_{2}$. We use as dimensionless control parameters the size asymmetry $\delta \equiv \sigma_{1} / \sigma_{2} \leq 1$ and either the two volume fractions $\phi_{1}$ and $\phi_{2}$ (with $\phi_{\alpha} \equiv \pi n_{\alpha} \sigma_{\alpha}^{3} / 6$ ), or the total packing fraction $\phi=\phi_{1}+\phi_{2}$ together with the molar fraction of the smaller species, $x_{1}=n_{1} /\left(n_{1}+n_{2}\right)$. This system serves to illustrate some features associated precisely with the size disparity. As discussed in more detail below, one expects that for mild asymmetries the only possible mode of dynamic arrest for all volume fractions and all molar fractions is that in which both species are arrested simultaneously. It is only in the regime of disparate sizes that one expects a richer scenario, including the second mode of dynamic arrest, in which the large particles are first arrested while the other species continues to diffuse, followed by a second transition at a higher total volume fraction in which the smaller particles are finally localized inside the pores of the matrix formed by the previously arrested particles. Thus, there must be a threshold asymmetry $\delta_{c}$ beyond which this might occur. One also expects, however, that even in the regime of considerable size disparities but for conditions corresponding to a few large particles in a see of smaller ones, the only mode of dynamic arrest continues to be

the mode of simultaneous dynamic arrest of the two species, since in this case the arrest of 
the small particles implies the arrest of the few large ones. We call this the "chancaquilla" limit [52], in reference to one of the many names of a rather universal sweet prepared by the vitrification of liquid molasses in which nuts or raisins are previously dispersed. Clearly, the dynamic arrest of the supporting molasses implies the arrest of the nuts dispersed in it. Hence, for large size asymmetries we expect to find both modes of dynamic arrest located in the neighborhood of the opposite composition limits, $x_{1}=0$ and $x_{1}=1$.

In fact, this is exactly the scenario predicted by our theory. We illustrate this with the SCGLE results obtained using the Percus-Yevick [53] structure factors as the static input, for a size asymmetry $\delta=0.3$. Let us calculate the collective and self propagator matrices $\Psi(t)$ and $\Psi^{(s)}(t)$ at increasing total volume fractions $\phi$ starting in the ergodic regime, crossing the dynamic arrest transition line, and ending inside the regime of arrested states. If this is done in the neighborhood of the $x_{1}=1$ axis, say for $x_{1}=0.8$, the scenario would be essentially that described by the chancaquilla mechanism involving the simultaneous dynamic arrest of both species, with similar results to those discussed in Fig. 2 for the Yukawa mixture. The other mode of dynamic arrest is observed in the opposite regime, where the small particles are a minority, exemplified by the molar fraction $x_{1}=0.2$. This case is illustrated in Fig. 3, which presents the evolution of the relaxation of the diagonal elements of the collective and self propagator matrices $\Psi(t)$ and $\Psi^{(s)}(t)$ as we increase the total volume fraction $\phi$ at fixed molar fraction $x_{1}=0.2$, starting in the ergodic regime. We find that, rather than passing directly from the fully ergodic region to the region of fully arrested states, as in Fig. 2. one first passes through an intermediate region of hybrid states, in which only the big particles are arrested but the small particles continue to diffuse. Thus, at a volume fraction $\phi_{g}^{(1)} \approx 0.545$, the system passes from the fully ergodic region to the region $\phi_{g}^{(1)}<\phi<\phi_{g}^{(2)}$ of hybrid states, with $\phi_{g}^{(2)}$ being the location of the second transition consisting of the localization of the small particles in the pores of the random matrix of big particles. In the present example this occurs at $\phi_{g}^{(2)} \approx 0.555$.

Let us mention that in all these cases one observes that the diagonal collective diffusion propagator $\Psi_{\alpha \alpha}(k, t)$ behave in qualitatively identical manner to the corresponding self diffusion propagator $\Psi_{\alpha \alpha}^{(s)}(k, t)$ in the sense that either both relax to zero or both exhibit dynamic arrest (i.e., decay to a finite value). One does not encounter a situation in which, for example, $\Psi_{11}(k, t)$ decays to a finite value while $\Psi_{11}^{(s)}(k, t)$ decays to zero. This indicates that at the level of the diagonal propagators, self and collective diffusion provide consistent 
descriptions of the pattern of relaxation. Thus, we may identify the fully ergodic states by the fact that all the elements of the (self and collective) propagators decay to zero, whereas the fully non-ergodic states can be identified by the decay of all the propagators to finite asymptotic values (identified with the non-ergodic parameters $\psi$ and $\psi^{s}$ ). The results for $\phi=0.53$ in Fig. [3 are representative of fully ergodic states, whereas those for $\phi=0.57$ illustrate fully arrested states. Hybrid states are characterized by a mixed behavior, in which the diagonal propagators $\Psi_{11}(k, t)$ and $\Psi_{11}^{(s)}(k, t)$ associated to the mobile species, decay to zero, while the diagonal propagators $\Psi_{22}(k, t)$ and $\Psi_{22}^{(s)}(k, t)$, associated to the large particles, exhibit arrested behavior. These conditions are illustrated in Fig. 3 by the results corresponding to $\phi=0.55$.

The relaxation of the crossed collective propagators $\Psi_{12}(k, t)$ and $\Psi_{21}(k, t)$, not shown in Fig. 3, do deserve some discussion, particularly in connection with mixed states. First, one must have in mind that, although the matrices $F(k, t)$ and $S(k)$ are symmetric, their product $F(k, t) S^{-1}(k)=\Psi(k, t)$ in general is not; thus, in general, $\Psi_{12}(k, t) \neq \Psi_{21}(k, t)$. Second, since the initial value $\Psi(k, 0)$ of the propagator matrix is the unit matrix, the initial value of the off-diagonal collective propagators $\Psi_{12}(k, t)$ and $\Psi_{21}(k, t)$ must vanish. Third, the off-diagonal collective propagators are not necessarily positive. This is illustrated in Fig. 4, which exhibits the time-relaxation of the four collective propagators $\Psi_{\alpha \beta}(k, t)$ for the same mixed state included in Fig. 3, but now evaluated at a common wave-vector $k$. The wave-vector employed in this figure corresponds to the position of the maximum of $S_{22}(k)$; for reference, the inset of this figure contains the partial static structure factors of this system. At this wave-vector we observe that both, $\Psi_{12}(k, t)$ and $\Psi_{21}(k, t)$, are negative, and that $\Psi_{12}(k, t)$ relaxes to a finite non-ergodic asymptotic value, while $\Psi_{21}(k, t)$ is always much smaller and relaxes to zero.

Let us mention that one could also display the same information not in terms of propagators but in terms of the so-called collective "correlators" $\Phi_{\alpha \beta}(k, t)$, defined as $\Phi_{\alpha \beta}(k, t) \equiv F_{\alpha \beta}(k, t) / S_{\alpha \beta}(k)$. Thus defined, the diagonal collective correlator $\Phi_{\alpha \alpha}(k, t)$ is a linear combination of the corresponding diagonal collective propagator $\Psi_{\alpha \alpha}(k, t)$ and of a crossed propagator; more precisely, $\Phi_{11}(k, t)=\Psi_{11}(k, t)+\left[S_{21}(k) / S_{11}(k)\right] \Psi_{12}(k, t)$ and $\Phi_{22}(k, t)=\Psi_{22}(k, t)+\left[S_{12}(k) / S_{22}(k)\right] \Psi_{21}(k, t)$. In the latter case, since $\Psi_{21}(k, t)$ is small and relaxes to zero, we find that the collective correlator $\Phi_{22}(k, t)$ is virtually identical to the collective propagator $\Psi_{22}(k, t)$, as shown in Fig. 4. In contrast, although the propagator 
$\Psi_{11}(k, t)$ does decay to zero, the diagonal collective correlator $\Phi_{11}(k, t)$ exhibits dynamic arrest due to its linear dependence on $\Psi_{12}(k, t)$ which, as illustrated in the figure, relaxes to a non-zero value. This observation may be relevant when describing the hybrid states above. For example, the description in terms of correlators has been employed in recent work [30, 36, 42], where the situation illustrated in Fig. 4 arises, leading to the notion that collective diffusion in mixtures is intrinsically different from self-diffusion. Our results indicate, however, that this apparent difference is just the result of the normalization convention employed in the definition of the correlators, and that if the same information were displayed in terms of the elements of the propagator matrices, no mixing of relaxation modes would occur that suggest this notion. We should stress that the self correlators, defined as $\Phi_{\alpha \beta}^{(s)}(k, t) \equiv F_{\alpha \beta}^{(s)}(k, t)$, are identical to the self propagators $\Phi_{\alpha \beta}^{(s)}(k, t)$, and hence correctly describe the fact that only the small particles diffuse and the large particles are arrested.

Finally, let us mention that distinguishing the three dynamic states above (fully ergodic, fully non-ergodic, and hybrid states) with only the information of the type in Figs. 3 and 4 is not a simple matter. The reason is that the non-ergodic parameters of the propagators associated with the localization of the small spheres do not jump discontinuously from zero to a finite appreciable value at $\phi_{g}^{(2)}$. Instead, they increase continuously from a value of zero right at this transition, and hence, attain finite but very small values in the close neighborhood of the transition. In the following section, however, we shall derive a simpler procedure to precisely locate these transitions and to evaluate the non-ergodic parameters in the (partially or totally) arrested states.

\section{FIXED-POINT EQUATIONS}

With the information just presented, one may choose to analyze the detailed timedependence of the propagators, and discuss the various regimes of the relaxation processes described by these functions at specific points in the state space. Alternatively, one can discuss more global properties, such as the general topology of the ergodic-nonergodic phase diagram of the system, just like one does in equilibrium statistical thermodynamics. In fact, the ergodic-nonergodic phase diagram should be an important complement to conventional equilibrium phase diagrams in terms of practical applications. If one is interested specifically in such global properties, in principle one does not need to solve the set of coupled 
equations (2.1) $-(2.6)$ for the full time- and wave-vector-dependence of all the dynamic properties involved. Instead, one may solve only the so-called fixed-point equations, i.e., the equations for the long-time asymptotic stationary solutions of the full SCGLE theory. The solutions thus determined are precisely the non-ergodic parameters, whose zero or non-zero value reveal if the state of the system is ergodic or dynamically arrested.

In order to derive the fixed point equations, let us define the non-ergodic parameter of the dynamic properties $\Psi(t), \Psi^{s}(t), C(t), C^{s}(t)$, and $\Delta \zeta^{*}(t)$, as the matrices $\psi(k) \equiv$ $\lim _{t \rightarrow \infty} \Psi(k, t), \psi^{s}(k) \equiv \lim _{t \rightarrow \infty} \Psi^{s}(k, t), c(k) \equiv \lim _{t \rightarrow \infty} C(k, t), c^{s}(k) \equiv \lim _{t \rightarrow \infty} C^{s}(k, t)$, and $\Delta \zeta^{*(\infty)} \equiv \lim _{t \rightarrow \infty} \Delta \zeta^{*}(t)$, respectively. All the elements of these matrices are zero in a fully ergodic state, and the non-zero value of some or all of them indicate partial or total non-ergodicity. Let us now substitute the dynamic properties involved in the self-consistent system of equations in Eqs. (2.1)-(2.6) by the sum of their asymptotic long-time value above, plus the rest (which, by definition, always relaxes to zero). In the resulting system of equations, let us then take the asymptotic long-time limit, thus generating the following self-consistent system of equations for the non-ergodic parameters

$$
\begin{gathered}
\psi(k)=\left[c(k)+k^{2} D S^{-1}(k)\right]^{-1} c(k), \\
\psi^{s}(k)=\left[c^{s}(k)+k^{2} D\right]^{-1} c^{s}(k), \\
c(k)=c^{s}(k) \\
c^{s}(k)=\lambda(k) \Delta \zeta^{*(\infty)}
\end{gathered}
$$

and

$$
\Delta \zeta_{\alpha}^{*(\infty)}=\frac{D_{\alpha}^{0}}{3(2 \pi)^{3}} \int d^{3} k k^{2}\left[\psi^{(s)}\right]_{\alpha \alpha}[c \sqrt{n} \psi \sqrt{n} h]_{\alpha \alpha},
$$

where the matrices $c$ and $h$ inside the integral in the last equation refer to the direct and total Ornstein-Zernike correlation functions, and together with the matrix $\sqrt{n}$, were defined below Eq. (2.6). Using Eqs. (5.3) and (15.4) in Eqs. (15.1) and (15.2), we can express the non-ergodic parameters $\psi(k)$ and $\psi_{S}(k)$ in terms of $\Delta \zeta^{*(\infty)}$. Substituting the resulting expressions in Eq. (5.5), we finally get the following closed equation for $\Delta \zeta^{*(\infty)}$ 


$$
\begin{aligned}
\Delta \zeta_{\alpha}^{*(\infty)}= & \frac{D_{\alpha}^{0}}{3(2 \pi)^{3}} \int d^{3} k k^{2}\left[\left(\lambda(k)+k^{2}\left(\Delta \zeta^{*(\infty)}\right)^{-1} D\right)^{-1} \lambda(k)\right]_{\alpha \alpha} \times \\
& \left\{c \sqrt{n}\left[\left(\lambda(k)+k^{2}\left(\Delta \zeta^{*(\infty)}\right)^{-1} D S^{-1}(k)\right)^{-1} \lambda(k)\right] \sqrt{n} h\right\}_{\alpha \alpha},
\end{aligned}
$$

for $\alpha=1,2, \ldots, \nu$, with $\nu$ being the number of species. Clearly, this equation always admits the trivial solutions $\Delta \zeta_{\alpha}^{*(\infty)}=0$, which corresponds to the fully ergodic fluid state. The existence of other non-zero real solution(s) is associated to the existence of arrested states.

It is convenient to re-write Eq. (5.6) in terms of the diagonal matrix $\gamma \equiv\left(\Delta \zeta^{*(\infty)}\right)^{-1} D$, whose diagonal elements $\gamma_{\alpha}$ will then satisfy the following set of equations

$$
\frac{1}{\gamma_{\alpha}}=\frac{1}{3(2 \pi)^{3}} \int d^{3} k k^{2}\left\{\left[I+k^{2} \gamma \lambda^{-1}(k)\right]^{-1}\right\}_{\alpha \alpha}\left\{c \sqrt{n}\left[I+k^{2} \gamma \lambda^{-1}(k) S^{-1}(k)\right]^{-1} \sqrt{n} h\right\}_{\alpha \alpha} .
$$

For the same reasons given in the mono-component case [17, 18], the new order parameters $\gamma_{\alpha}$ may be identified with the long-time asymptotic value of the mean squared displacement (msd) of particles of species $\alpha$. Thus, $\gamma_{\alpha}$ is infinite if that species diffuses, and is finite if it is arrested; in fact, in the latter case $\gamma_{\alpha}^{1 / 2}$ is the localization length of the particles of that arrested species. In terms of $\gamma$, Eqs. (5.1) and (5.2) for the non-ergodic parameters $\psi(k)$ and $\psi^{s}(k)$ read

$$
\psi(k)=\left[I+k^{2} \gamma \lambda^{-1}(k) S^{-1}(k)\right]^{-1}
$$

and

$$
\psi^{s}(k)=\left[I+k^{2} \gamma \lambda^{-1}(k)\right]^{-1} .
$$

Eqs. (5.7)-(5.9) are the fixed point equations of the SCGLE theory. In the particular case of a mono-disperse system, one recovers the result derived and employed in Refs. [17, 18]. For a given system, i.e., for given pair potentials, one is supposed to determine first the static structure factors $S_{\alpha \beta}(k)$, which is the only external input in Eq. (5.7) (recall that the matrix $\lambda(k)$ is determined by these structural properties, according to Eq. (2.4)). Eqs. (5.7) can be solved numerically, and the solutions for $\gamma$ are then employed in Eqs. (5.8) and (5.9) to determine the non-ergodic parameters. 
Let us apply Eqs. (5.7) to calculate the order parameters $\gamma_{1}$ and $\gamma_{2}$ for binary hard-sphere mixtures. The corresponding partial static structure factors $S_{\alpha \beta}(k)$ needed in that equation will again be provided by the Percus-Yevick (PY) approximation. From the resulting values of $\gamma_{1}$ and $\gamma_{2}$, we can classify the states of this system as ergodic, fully non-ergodic, or partially non-ergodic, and thus scan the state space to determine the regions of these dynamic phases and the boundaries between them. For this, let us adopt the asymmetry parameter $\delta$, the total volume fraction $\phi$, and the molar fraction $x_{1}$ of the smaller spheres as the dimensionless state variables spanning the state space $\left(\delta, \phi, x_{1}\right)$.

Before presenting the results of this exercise, that we collect in Fig. 5, let us say that from intuitive arguments one may advance some of the features of this phase diagram. For example, as already indicated in section IV one expects that for mild asymmetries the only possible mode of dynamic arrest for all volume fractions and all molar fractions is that in which both species are arrested simultaneously. The reason for this expectation is that this regime must contain the extreme case corresponding to the degenerate limit $\delta=1$. From the point of view of their interactions, in this limit the particles of both species are actually indistinguishable $\left(\sigma_{1}=\sigma_{2}\right)$, thus being in fact a mono-disperse system, whose particles have been classified only artificially as belonging to either of these two species. We expect, then, that for any molar fraction $0 \leq x_{1} \leq 1$, the system will be arrested at the total volume fraction $\phi_{g}^{(m)}$ at which a mono-disperse system would be arrested. This means that in the plane $\left(\delta=1, \phi, x_{1}\right)$, the transition line must correspond to the condition $\phi_{1}+\phi_{2}=\phi_{g}=\phi_{g}^{(m)}$. According to the mono-component version of the SCGLE theory, and within the same level of approximation adopted here for the static structure factor (i.e., the PY approximation) we know that $\phi_{g}^{(m)}=0.537$ [18]. Thus, in the degenerate case the straight line $\phi=\phi_{g}^{(m)}$ (horizontal line in Fig. 5. a) separates the ergodic region $\phi<\phi_{g}^{(m)}$ from the region $\phi>\phi_{g}^{(m)}$ of totally non-ergodic states, in which both species are dynamically arrested.

Thus, the first question is now if the SCGLE theory has built-in this degenerate limit, i.e., if under these degenerate conditions, Eqs. (5.7)-(5.9) reduce to the mono-disperse result discussed in references [17, 18]. Indeed, if one constrains the solution for $\gamma_{1}$ and $\gamma_{2}$ to exhibit this symmetry, i.e., that $\gamma_{1}=\gamma_{2}$, one can show that the two equations involved in Eq. (5.7) are indeed identical to each other and to the corresponding mono-disperse equation, independently of the composition represented by the molar fraction $x_{1}$. This means that both "species" either remain ergodic or are arrested together. 
The next question is then what happens when this degeneration is broken, so that $\sigma_{1} \leq \sigma_{2}$, but remaining in the region of mild asymmetry $\delta \lesssim 1$, in which we expect small deviations from the degenerate limit. Unfortunately, we do not have good intuitive arguments to anticipate even the sign of these deviations. In Fig. 5. a, however, we report the predictions of our theory for this regime, in terms of the glass transition lines corresponding to the values of the asymmetry parameter $\delta=1.0,0.8,0.6$ and 0.4 , for which our theory predicts that the glass transition line moves to higher total volume fractions $\phi_{g}$ as the size disparity increases (i.e., $\delta$ decreases), except at the two ends $x_{1}=0$ and $x_{1}=1$ of the transition line, where the value of $\phi_{g}$ must be fixed by its mono-disperse value $\phi_{g}^{(m)}$ for all size disparities.

The general trends illustrated by Fig. 5. a were actually first observed and described by Williams and van Megen [29] in their experimental process of melting an originally monodisperse glass by the replacement of a fraction of its particles by particles of a smaller size keeping the same total volume fraction. The dots and the arrow in Fig. 5, a correspond to their experimental process, that may conceptually be described as driving a system from the initially mono-disperse glass state $\left(\delta, \phi / \phi_{g}^{(m)}, x_{1}\right)=(0.6,1.0175,0.0)$ through the arrested states $(0.6,1.0175,0.196)$ and $(0.6,1.0175,0.340)$, to end in the ergodic state $(0.6,1.0175,0.537)$ which lies in the ergodic region according to our predictions for the same size asymmetry $\delta=0.6$ of the experimental system (the representation of this process in the scale of Fig. 5. a involves the re-scaling of the experimental volume fractions $\phi^{\exp }=$ 0.58 with the experimental value of $\phi_{g}^{(m)}$ which, within the experimental errors, we took as $\left.\phi_{g}^{(m)}=0.57\right)$.

The regime illustrated in Fig. 57a was also studied by Götze and Voigtmann with MCT [37, 38], with predictions qualitatively similar to ours for intermediate size-disparities $(\delta \lesssim$ 0.65), but with conflicting predictions for milder size disparities $(\delta \sim 0.8)$, where MCT predicts "S-shaped" transition lines that may go below the mono-disperse glass transition volume fraction $\phi_{g}^{(m)}$ [37]. The SCGLE theory, predicts, instead, that the transition lines for the mixture, as illustrated in Fig. 5, a always lie above the horizontal transition line corresponding to the degenerate mono-disperse case. These conflicting predictions of MCT and the SCGLE theory could be resolved by experiments and/or computer simulations. The actual magnitude of the difference between the predictions of the two theories is, however, so small that a conclusive discrimination between them will require experimental or simulation results of higher resolution than provided by the currently available data [7, 39]. 
Fig. 5,b illustrates the regime of large size disparities. The SCGLE theory predicts that below a threshold asymmetry $\delta_{c} \sim 0.4$, a region of mixed states appear, which is illustrated by the shaded area in the results for $\delta=0.3$. This region is bounded on the left by the axis $x_{1}=0$, below by the transition of dynamic arrest of the large particles (solid line), and above by the localization transition of the small particles (dashed line). The region of mixed states ends on the right in the bifurcation point where these two transition lines meet, and where the transition of simultaneous dynamic arrest of both species starts, ending in the axis $x_{1}=1$ (solid line). The area of the shaded region increases as $\delta$ decreases, as illustrated by the results for $\delta=0.2$, in which the region of mixed states was not shaded. Of course, as $\delta$ approaches the threshold value $\delta_{c} \sim 0.4$, the bifurcation point moves to the axis $x_{1}=0$, and the region of mixed states disappears. Although we cannot in practice explore the limit $\delta<<1$, we do not expect additional qualitatively different trends beside those exhibited by the illustrative cases in Fig. 5. Thus, to our knowledge, this is the first time that the entire ergodic-non-ergodic phase diagram of a binary colloidal mixture has been outlined.

\section{CONCLUDING REMARKS}

In this paper we have introduced the self-consistent generalized Langevin equation theory of dynamic arrest in colloidal mixtures, and we have applied it to the description of dynamic arrest phenomena in two simple model systems, namely, a repulsive Yukawa and a hardsphere mixtures, for which we illustrated the scenarios predicted by this theory through the full numerical solution of the coupled system of dynamic equations for the propagators $\Psi(t)$ and $\Psi^{(s)}(t)$ of the self and collective intermediate scattering functions. In this manner we identified and illustrated the two possible patterns of dynamic arrest in a binary colloidal mixture, namely, the simultaneous and the sequential arrest of the two species. The for-

mer corresponds to the transition from the region of fully ergodic states, characterized by infinite values of the order parameters $\gamma_{1}$ and $\gamma_{2}$, to the region of fully non-ergodic states, characterized by finite values of these two parameters. This transition is characterized by a discontinuous jump of both order parameters. This is the only dynamic arrest transition possible for moderate size asymmetry in a binary hard-sphere mixture, as illustrated in Fig. 2. It is also the only transition possible for large asymmetries in the case of hard spheres provided the system is in the limit of a few large particles dispersed in a sea of small particles 
(the dynamic arrest of "chancaquilla" limit).

The second pattern of dynamic arrest is only observed for large enough size disparities starting in the regime in which the small particles are a minority. As illustrated in Fig. 3, here the system may be driven from the region of fully ergodic states $\left(\gamma_{1}=\gamma_{2}=\infty\right)$ to the region of mixed states (finite $\gamma_{2}$ and $\gamma_{1}=\infty$ ) in which the large particles are arrested and the small ones diffuse through the voids left by the large species. In this transition $\gamma_{2}$ jumps discontinuously from its infinite value in the fully non-ergodic region to a finite value in the region of mixed states, whereas $\gamma_{1}$ retains its infinite value. Finally, there is a transition from this region of mixed states to the region of fully ergodic states in which also the small particles are localized (finite values of both, $\gamma_{1}$ and $\gamma_{2}$ ). This latter transition is characterized by a continuous change in the values of both order parameters $\gamma_{1}$ and $\gamma_{2}$, although the latter changes continuously from its infinite value inside the region of mixed states and right at the transition, to finite values inside the fully non-ergodic region.

Locating the transitions just described may be done by numerically solving the full selfconsistent system of equation, as done in Figs. 2 and 3, or by directly determining the nonergodic parameters, which play the role of order parameters of the transition. The latter can be done more economically through the solution of the "fixed-point" equations. In section IV we derived a strikingly simple and general result for the non-ergodic parameters $\gamma_{1}$ and $\gamma_{2}$, whose use was illustrated with the construction of the phase diagram of the hard-sphere binary mixture. We must say that some of the features of the SCGLE results thus determined were in fact discovered and discussed in the pioneering work of Refs. [32, 34, 35, 36] based on the multi-component version of MCT. Our aim here has been to document what is the scenario offered by the SCGLE theory for the same conditions previously studied, and to extend the description to new aspects not yet discussed with MCT or with other theoretical approaches. In this regard, the main contribution of the present work is the proposal of a new theoretical approach that allows the determination of the entire dynamic phase diagram of simple model colloidal mixtures. Thus, the second important contribution involves the simplest of such models, namely, the hard-sphere binary mixture, for which we outlined the entire dynamic arrest phase diagram. In this context we discussed the regime of mild size asymmetries, in which we reported the first apparent difference with the results of MCT, namely, the fact that the SCGLE theory does not predict the S-shaped transitions predicted by Götze and Voigtmann [37] on the basis of MCT. For more severe size disparities we also 
illustrated the main features of the SCGLE predictions, including the precise location of the region of mixed states, and its emergence for size disparities beyond a threshold asymmetry $\delta_{c} \approx 0.4$. These results do not have yet a MCT counterpart to compare with.

In this paper we also discussed the fact that other properties besides $\gamma_{1}$ and $\gamma_{2}$ could be employed as order parameters to classify the various dynamic phases in a mixture. For example, one can use the diagonal elements of the non-ergodic parameters associated with the self or the collective propagator matrices, which also determine unambiguously the various possible states. Although not central to the main subject of this paper, we also explained that the use as order parameters of collective correlators, defined as the partial intermediate scattering functions divided by their initial value, may lead to a confusing description of the hybrid states in which one species is arrested and the other remains ergodic.

In summary, we have presented a theory for dynamic arrest phenomena in colloidal mixtures that offers some advantages over the extension to mixtures of conventional MCT. First, it derives from a simpler conceptual formalism, which might allow possible generalizations and extensions. Second, the resulting equations are much simpler to solve in practice than the corresponding equations of MCT. In fact, the determination of (ergodic-non-ergodic) phase diagrams, such as that discussed in the previous section, is considerably simplified in the present case through the use of the results for the non-ergodic parameters $\gamma_{\alpha}$ in Eq. (5.7). Thus, many important issues regarding the dynamics and the (ergodic-non-ergodic) phase behavior of colloidal mixtures may be discussed with the assistance of the SCGLE theory just presented. The implications of the results presented here in the context of specific physical phenomena and experimental conditions will, however, be the subject of separate reports.

\section{ACKNOWLEDGMENTS:}

This work was supported by the Consejo Nacional de Ciencia y Tecnología (CONACYT, México), through grant No. SEP-2004-C01-47611 and of FAI-UASLP. The authors are 
deeply indebted to Profs. J. Bergenholtz, A. Banchio, and G. Nägele for useful discussions.

[1] C. A. Angell, Science 267, 1924 (1995).

[2] P. G. Debenedetti and F. H. Stillinger, Nature 410, 359 (2001).

[3] W. Götze, in Liquids, Freezing and Glass Transition, edited by J. P. Hansen, D. Levesque, and J. Zinn-Justin (North-Holland, Amsterdam, 1991).

[4] P. N. Pusey, in Liquids, Freezing and the Glass transition, edited by J. P. Hansen, D. Levesque, and J. Zinn-Justin (Elsevier, Amsterdam, 1991).

[5] W. Hess and R. Klein, Adv. Phys. 32, 173 (1983).

[6] G. Nägele, Phys. Rep. 272, 215 (1996).

[7] W. van Megen and P. N. Pusey, Phys. Rev. A 43, 5429 (1991).

[8] E. Bartsch et al., J. Chem. Phys. 106, 3743 (1997).

[9] C. Beck. W. Härtl, and R. Hempelmann, J. Chem. Phys. 111, 8209 (1999).

[10] K. N. Pham et al., Science, 296, 104 (2002).

[11] F. Sciortino and P. Tartaglia, Adv. Phys. 54, 471 (2005).

[12] W. Götze and L. Sjögren, Rep. Prog. Phys. 55, 241 (1992).

[13] G. Szamel, Phys. Rev. Lett. 90, 228301 (2003).

[14] J. Wu and J. Cao, Phys. Rev. Lett. 95, 078301 (2005).

[15] P. Mayer, K. Miyazaki, and D. R. Reichman, Phys. Rev. Lett. 97, 095702 (2006).

[16] M. Tokuyama, Phys. Pev. E 62, R5915 (2000).

[17] P. Ramírez-González, R. Juárez-Maldonado, L. Yeomans-Reyna, M. A. Chávez-Rojo, M. Chávez-Páez, A. Vizcarra-Rendón, and M. Medina-Noyola, Rev. Mex. Física (in press, 2007).

[18] L. Yeomans-Reyna, M. A. Chávez-Rojo, P. E. Ramírez-González, R. Juárez-Maldonado, M. Chávez-Páez, and M. Medina-Noyola, Phys. Rev. E 76, 041504 (2007).

[19] R. Juárez-Maldonado, M. A. Chávez-Rojo, P. E. Ramírez-González, L. Yeomans-Reyna, and M. Medina-Noyola, submitted to Phys. Rev. E (2007).

[20] L. Yeomans-Reyna and M. Medina-Noyola, Phys. Rev. E 62, 3382 (2000).

[21] L. Yeomans-Reyna, H. Acuña-Campa, and M. Medina-Noyola, Phys. Rev. E 62, 3395 (2000).

[22] L. Yeomans-Reyna and M. Medina-Noyola, Phys. Rev. E 64, 066114 (2001).

[23] L. Yeomans-Reyna, H. Acuña-Campa, F. Guevara-Rodríguez, and M. Medina-Noyola, Phys. 
Rev. E 67, 021108 (2003).

[24] M. A. Chávez-Rojo and M. Medina-Noyola, Physica A 366, 55 (2006).

[25] M. A. Chávez-Rojo and M. Medina-Noyola, Phys. Rev. E 72, 031107 (2005); ibid 76, 039902 (2007).

[26] A. Meller and J. Stavans, Phys. Rev. Lett. 68, 3646 (1992).

[27] A. Imhof and J. K. G. Dhont, Phys. Rev. Lett. 75, 1662 (1995).

[28] A. Imhof and J. K. G. Dhont, Phys. Rev. E 52, 6344 (1995).

[29] S. R. Williams and W. van Megen, Phys. Rev. E 64, 041502 (2001).

[30] A. J. Moreno and J. Colmenero, J. Chem. Phys. 125, 164507 (2006).

[31] N. Kikuchi and J. Orbach, Europhys. Lett. 77, 26001 (2007).

[32] J. Bosse and J. S. Thakur, Phys. Rev. Lett. 59, 998 (1987).

[33] J. L. Barrat and A. Latz, J. Phys. Condens. Matter 2, 4289 (1990).

[34] J. S. Thakur and J. Bosse, Phys. Rev. A 43, 4378 (1991); ibid. 43, 4388 (1991).

[35] J. Bosse and Y. Kaneko, Phys. Rev. Lett. 74, 4023 (1995).

[36] G. Nägele, J. Bergenholtz and J. K. G. Dhont, J. Chem. Phys. 110, 7037 (1999)

[37] W. Götze and Th. Voigtmann, Phys. Rev. E 67, 021502 (2003).

[38] Th. Voigtmann, Phys. Rev. E 68, 051401 (2003).

[39] G. Foffi, W. Götze, F.Sciortino, P. Tartaglia, and Th. Voigtmann, Phys. Rev. E 69, 011505 (2004); Phys. Rev. Lett. 91, 085701 (2003).

[40] Th. Voigtmann, A. M. Puertas, and M. Fuchs, Phys. Rev. E 70, 061506 (2004).

[41] E. Flenner and G. Szamel, Phys. Rev. E 72, 031508 (2005).

[42] Th. Voigtmann and J. Horbach, Europhys. Lett. 74, 459 (2006).

[43] E. Zaccarelli, H. Löwen, P. P. F. Wessels, F. Sciortino, P. Tartaglia, and C. N. Lykos, Phys. Rev. Lett., 92, 225703 (2004).

[44] In reality, Nägele et al. [36] refer to the matrix product $[C(k, z) D]$ as the matrix of "irreducible memory functions", a concept first introduced by B. Cichocki and W. Hess, Physica A 141, 475 (1987).

[45] J. L. Arauz-Lara and M. Medina-Noyola, Physica A 122, 547 (1983).

[46] M. Medina-Noyola, Faraday Discuss. Chem. Soc. 83, 21 (1987).

[47] M. Medina-Noyola and J. L. del Río-Correa, Physica A 146, 483 (1987).

[48] M. Hernandez-Contreras, M. Medina-Noyola, and A. Vizcarra-Rendon, Physica A 234, 271 
(1996).

[49] M. Fuchs, W. Götze, I. Hofacker, and A. Latz, J. Phys.: Condens. Matter 3, 5047 (1991).

[50] A. J. Banchio, Diffusion, Rheology and Polydispersity Effects in Three-dimensional and (Quasi)-two-dimensional Colloids, Ph.D. Thesis, University of Konstanz (1999).

[51] J. P. Hansen and I. R. McDonald, Theory of Simple Liquids (Academic Press Inc., 1976).

[52] Chancaquilla is one of the many names for one of the many versions of this candy, in this case made with pumpkin and sesame seeds in the Rio Verde region of the mexican state of San Luis Potosí.

[53] J. L. Lebowitz, Phys. Rev. 133, A895 (1964). 


\section{FIGURES AND CAPTIONS:}
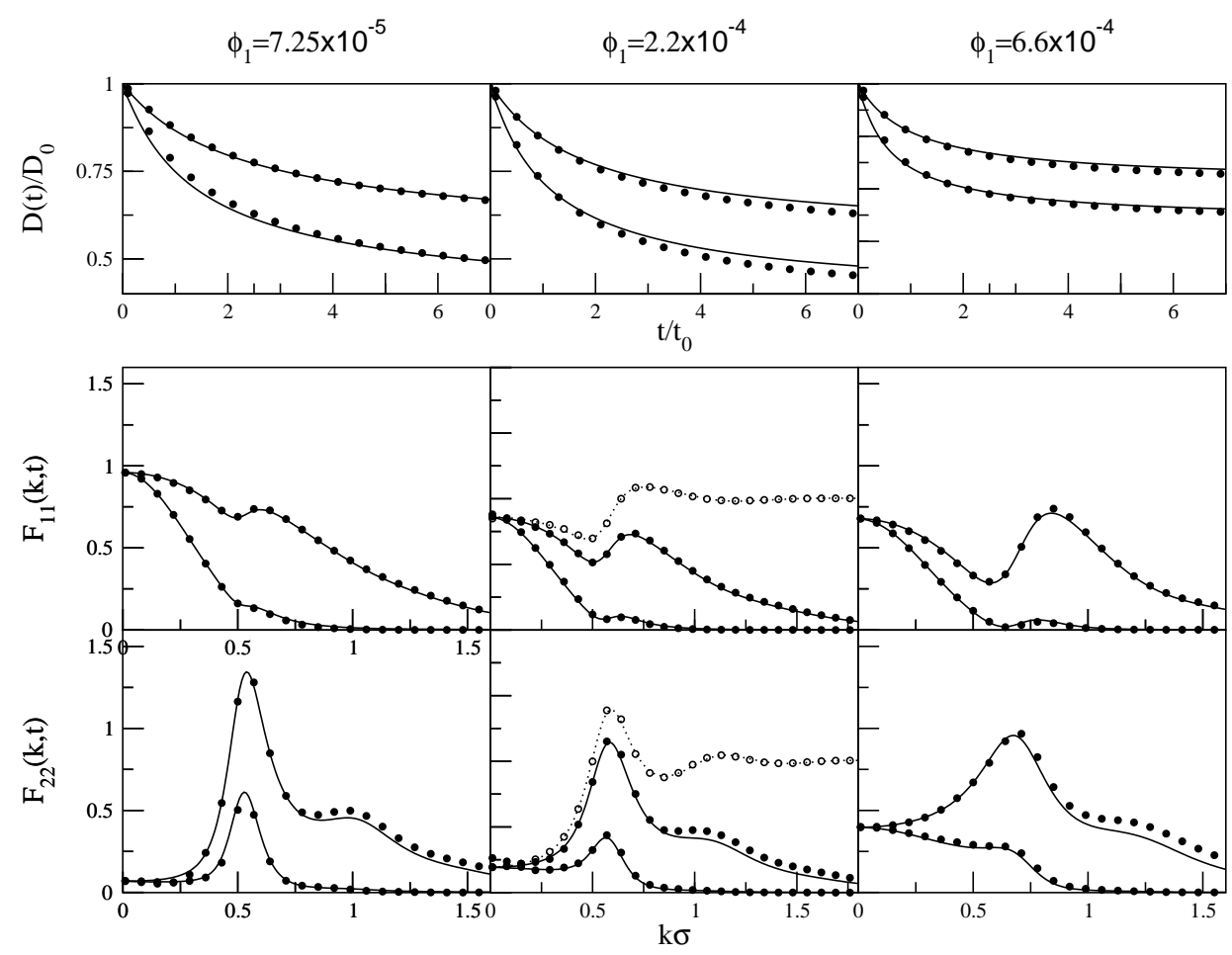

FIG. 1: Time-dependent diffusion coefficients $D_{\alpha}(t)$ as a function of time (upper curves corresponding to $\left.D_{1}(t)\right)$, and total intermediate scattering functions $F_{11}(k, t)$ and $F_{22}(k, t)$ as a function of wave-vector of a repulsive Yukawa mixture with $z=0.15, A_{1}=10, A_{2}=10 \sqrt{5}$ for $t=t_{0}$ and $t=10 t_{0}$. The volume fraction of the more interacting species is kept fixed at $\phi_{2}=2.2 \times 10^{-4}$ and $\phi_{1}$ takes the values $\phi_{1}=0.725 \times 10^{-4}$ (left column), $\phi_{1}=2.2 \times 10^{-4}$ (middle column), and $\phi_{1}=6.6 \times 10^{-4}$ (right column). Solid lines are the SCGLE theoretical results and circles are Brownian dynamics data. In the middle column we also include the partial static structure factors $S_{\alpha \alpha}(k) \equiv F_{\alpha \alpha}(k, t=0)$, with the dotted lines being a smooth HNC-fit of the simulation data. 


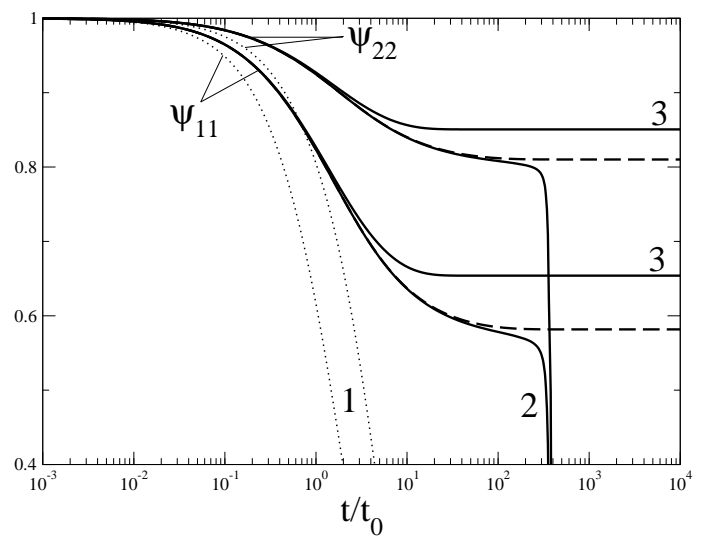

FIG. 2: SCGLE results for the collective diffusion propagators $\Psi_{\alpha \alpha}\left(k_{\max }, t\right)$ at fixed wave-vector $k_{\max }$ (the position of the main peak of $\left.S_{\alpha \alpha}(k)\right)$ for the Yukawa mixture with $z=0.15, \phi_{1}=\phi_{2}=$ $2.2 \times 10^{-4}$ calculated using HNC partial static structure factors evaluated at effective coupling parameters given by $A_{\alpha}=A_{\alpha}^{0} \chi$, with $A_{1}^{0}=10$ and $A_{2}^{0}=10 \sqrt{5}$, for values of $\chi$ corresponding to the reference state in Fig. $1\left(\chi^{(1)}=1.225\right.$, curves labeled " 1 " $)$ and to states slightly below $\left(\chi^{(2)}=4.255\right.$, labeled " 2 ") and above $\left(\chi^{(3)}=4.330\right.$, labeled "3"), the dynamic arrest transition. The dashed plateaus indicate the value of the non-ergodic parameters right at the transition $(\chi=4.257)$. 

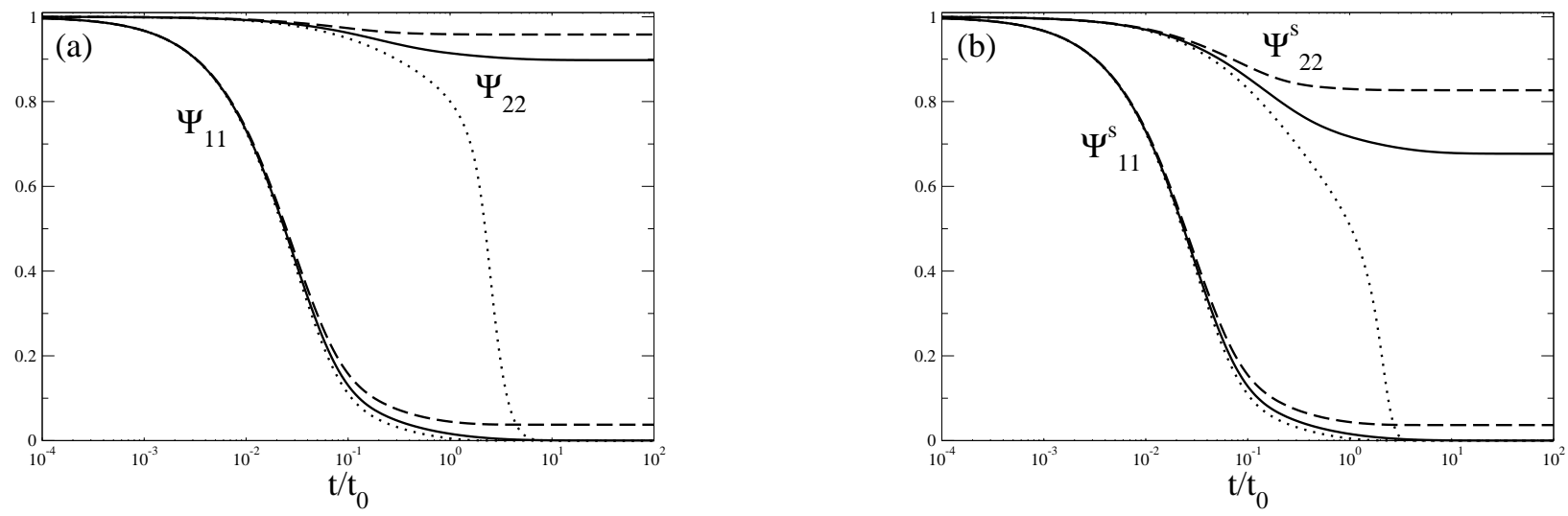

FIG. 3: (a) Collective diffusion propagators $\Psi_{\alpha \alpha}\left(k_{\max }, t\right)$ and (b) self diffusion propagators $\Psi_{\alpha \alpha}^{(s)}\left(k_{\max }, t\right)$ at fixed wave-vector $k_{\max }$ (the position of the main peak of $S_{\alpha \alpha}(k)$ ) for the hardsphere mixture with $\delta=0.3$ and $x_{1}=0.2$ for different total packing fraction $\phi$. The results for $\phi=0.53$ (dotted lines) illustrate the fully ergodic states, whereas those for $\phi=57$ (dashed lines) illustrate the fully arrested states, in which both species are arrested. The results for $\phi=0.55$ (solid lines) illustrate the hybrid states in which the large particles are arrested while the smaller particles continue to diffuse.

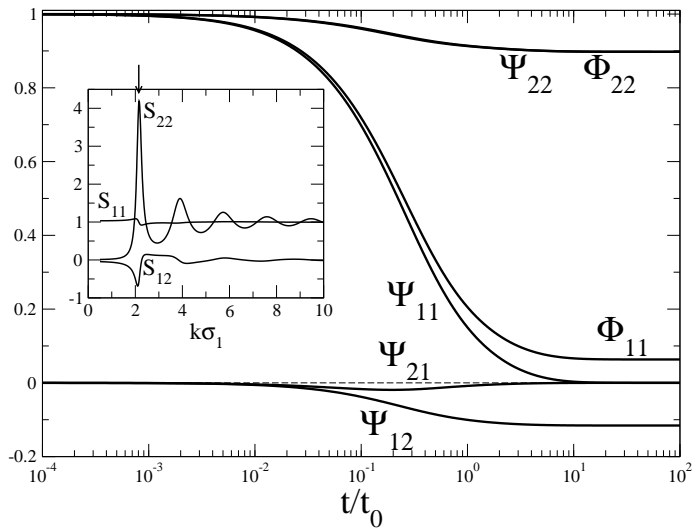

FIG. 4: Collective diffusion propagators $\Psi_{\alpha \beta}(k, t)$ and diagonal collective diffusion correlators $\Phi_{\alpha \alpha}(k, t)$ at a common wave-vector corresponding to the position of the main peak of $S_{22}(k)$ (see the inset, which contains static structure factors at this state) for the hard-sphere mixture with $\delta=0.3$ and $x_{1}=0.2$ for the hybrid state with $\phi=0.55$. 

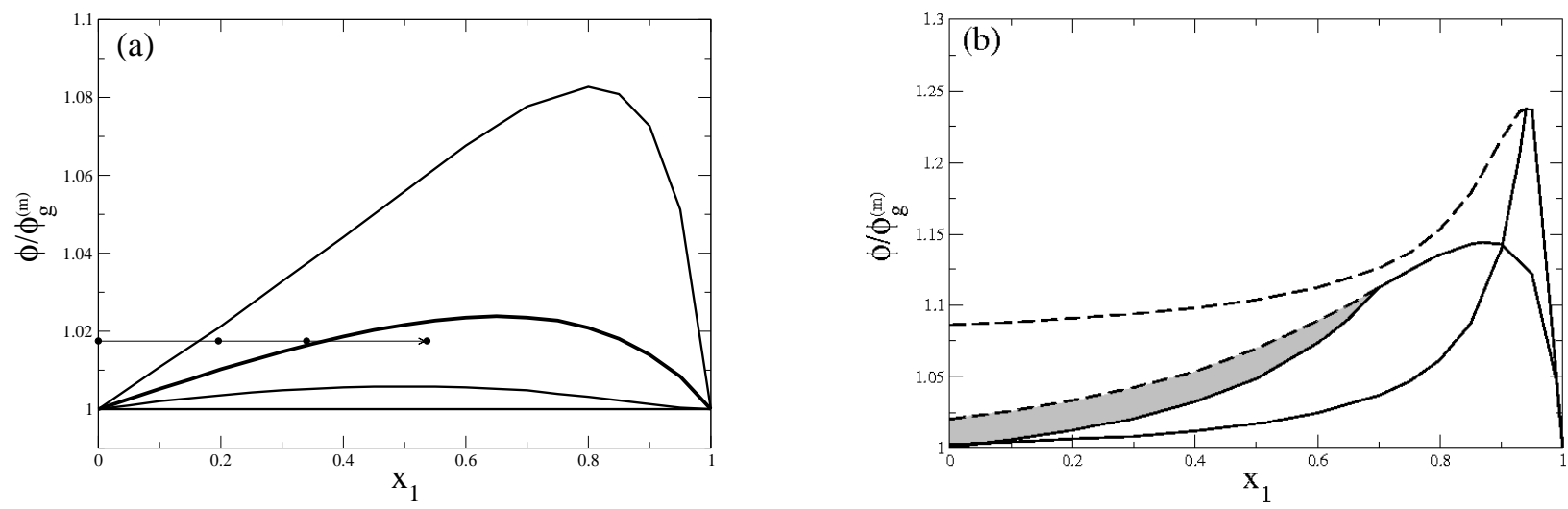

FIG. 5: Phase diagram of dynamic arrest states of the binary hard-sphere mixture. The threedimensional state space $\left(\delta, \phi, x_{1}\right)$ is spanned by the size disparity parameter $\delta \equiv \sigma_{1} / \sigma_{2} \leq 1$, the total volume fraction $\phi \equiv \phi_{1}+\phi_{2}$ with $\phi_{\alpha} \equiv \pi n_{\alpha} \sigma_{\alpha}^{3} / 6$, and the molar fraction of the smaller particles $x_{1} \equiv n_{1} /\left(n_{1}+n_{2}\right)$, where $\sigma_{\alpha}$ and $n_{\alpha}$ are the hard-core diameter and the number concentration of particles of species $\alpha$. This state space is partitioned in three regions, corresponding to fully ergodic, mixed, and fully non-ergodic states. In this figure we show the intersection lines of this transition surface with the planes of constant $\delta$. The total volume fraction $\phi$ has been scaled with the volume fraction $\phi_{g}^{(m)}$ of the ideal glass transition of the mono-disperse hard-sphere system. In (a) we illustrate the regime of mild size disparities with the values $\delta=1.0$ (horizontal line, corresponding to $\left.\phi=\phi_{g}^{(m)}\right), 0.8,0.6$ (thicker line), and 0.4 , where the respective line divides the subspace $\left(\phi, x_{1}\right)$ in the regions of fully ergodic states (below the line) and fully non-ergodic states. The four dots linked by the arrow at fixed total volume fraction $\phi / \phi_{g}^{(m)}=1.0175$ correspond to the states prepared in the experiment of Williams and van Megen (Ref. [29]) for $\delta=0.6$. In (b) we illustrate the regime of severe size-disparities with the values $\delta=0.3$ and 0.2 . In this regime the region of fully ergodic states also lies below the solid line but a region of mixed states appear (shaded region for the case $\delta=0.3$ ). This region is bounded from above by the localization transition of the small particles (dashed lines). 